\title{
Mode of Action and Cytotoxicity of Bioactive Compounds Produced by Streptomyces sp. KB1
}

\author{
Monthon Lertcanawanichakul ${ }^{1,2}$ and Kittisak Chawawisit ${ }^{1}$ \\ 1. School of Allied Health Sciences, Walailak University, Nakhon Si Thammarat 80160, Thailand \\ 2. Tropical Medicine Research Unit, Research Institute for Health Sciences, Walailak University, Nakhon Si Thammarat 80160, \\ Thailand
}

\begin{abstract}
Rapid emergence of methicillin-resistant Staphylococcus aureus (MRSA) has led to search of a novel bioactive compounds from natural resources. This study was aimed to determine the mode of action of bioactive compounds produced by Streptomyces sp. KB1 TISTR 2304 against MRSA, including cytotoxicity against mature brine shrimp. The mode of action and cytotoxicity of bioactive compounds were performed by observing the tested MRSA cells with a scanning electron microscope and evaluated by the brine shrimp lethality bioassay, respectively. The results indicated that bioactive compounds had the mode of action at the cell wall and also showed the moderate cytotoxic activity. This study concluded that the bioactive compounds produced from strain KB1 can be used as a model for novel anti-MRSA drug development.
\end{abstract}

Key words: Anti-MRSA activity, bioactive compounds, mode of action, cytotoxicity, Streptomyces sp. KB1 TISTR 2304.

\section{Introduction}

The problem of methicillin-resistant Staphylococcus aureus (MRSA), the greatest concern of all health-care-associated pathogen, resisted to the approved antibiotics that increased rapidly of several areas has become a worldwide problem with serious consequences of the treatment of MRSA infection. It is possibly due to its ability to cause a wide variety of life-threatening infections. MRSA has the ability to rapidly adapt to different environmental conditions [1-4]. Formerly, it was described as a nosocomial pathogen and a problem confined to institutionalized patients. More recently, spread of MRSA to the community setting has been described in injection drug users, prisoners and children. Today, MRSA has been recognized as one of the important pathogenic bacteria in community-acquired and hospital-acquired infections [3]. That the increased use/misuse of

Corresponding author: Monthon Lertcanawanichakul, Ph.D., associate professor, research fields: microbiology and biomedical sciences. antibiotic in a treatment of MRSA infectious disease is mainly caused to the MRSA develops a mechanism of antibiotic resistance [5, 6]. Such, new antibiotics will have to be developed in order to treat MRSA infections. New and more efficient antibiotics will have to be sought continually because of the capacity of microorganisms to survive their action. Many different strategies for finding new anti-MRSA compounds are actually proposed and the area of bioactive compounds (secondary metabolites) is under intense investigation. Among microorganisms, actinomycetes, especially the genus Streptomyces, are a prominent source of natural bioactive compounds that have important applications in human medicine. They are gram-positive bacteria known for their capacity to produce antibiotics and other medically important agents such as anti-cancer, anti-inflammatory, antifungal, antihelminthic and herbicide agents [5, 7]. Approximately, two thirds of all currently used antibiotics were developed from their bioactive compounds [8]. Streptomyces sp. KB1 could produce bioactive compounds and excrete into 
the liquid culture medium. These bioactive compounds in culture broth were extracted with ethyl acetate solvent. The preliminarily data showed that partially purified bioactive compounds had the anti-MRSA activity when tested by agar well diffusion method. Therefore, the purpose of this study was to determine the mode of action of bioactive compounds against clinical isolates of MRSA, along with evaluating its cytotoxicity against the mature brine shrimp.

\section{Method and Materials}

\subsection{Microorganisms, Media and Culture Conditions}

The Streptomyces sp. KB1 TISTR 2304 was collected from air at Aonang, Krabi province by using air bio-sampler (Microflow 90) at a flow rate 100 $\mathrm{L} / \mathrm{min}$ for $30 \mathrm{~min}$. It was used as a source of bioactive compounds. The partially purified bioactive compounds were extracted from culture broth of strain KB1 with ethyl acetate solvent in a ratio 1:1, dried using rotary evaporator apparatus. It was kept at $-20^{\circ} \mathrm{C}$ until use.

The MRSA clinical isolate of 2468 (MRSA 2468) was kindly obtained from the Maharaj Nakhon Si Thammarat Hospital, Thailand. Methicillin sensitive Staphylococcus aureus (MSSA) TISTR 517 was purchased from Thailand Institute of Scientific and Technological Research (TISTR), Thailand. Both MRSA and MSSA were cultured in Luria Bertani (LB; Himedia, India) agar medium at $37{ }^{\circ} \mathrm{C}$ in static incubator for $24 \mathrm{~h}$. Single colony of each isolate was inoculated into $10 \mathrm{~mL}$ of $\mathrm{LB}$ broth medium in $25 \times$ $150 \mathrm{~mm}$ of the screw cap test tube, incubated at $37^{\circ} \mathrm{C}$, $200 \mathrm{rpm}$ in shaking incubator for $24 \mathrm{~h}$ and stored in $15 \%$ glycerol at $-80{ }^{\circ} \mathrm{C}$ until use.

\subsection{Mode of Action of Bioactive Compounds}

Mode of action of bioactive compounds was performed by applying from published protocol [8]. Single colony of MRSA 2468 was inoculated into 10 $\mathrm{mL}$ of Mueller-Hinton (M-H) broth medium in $25 \times$ $150 \mathrm{~mm}$ of the screw cap test tube, incubated at $37^{\circ} \mathrm{C}$,
$200 \mathrm{rpm}$ in shaking incubator for $24 \mathrm{~h}$. The cell suspension was inoculated into $50 \mathrm{~mL}$ of M-H broth medium in $250 \mathrm{~mL}$ of Duran bottle to obtain a final concentration of $1.5 \times 10^{8} \mathrm{CFU} / \mathrm{mL}$ or turbidity comparable to 0.5 McFarland standards, added the bioactive compounds and designed as the experimental group. MRSA cell suspension without bioactive compound was designed as a control group. Both experimental and control groups were incubated at $37^{\circ} \mathrm{C}, 200 \mathrm{rpm}$ in shaking incubator for $24 \mathrm{~h}$. Every $8 \mathrm{~h}$, the cell suspension was harvested and centrifuged to separate the cells sediment and supernatant at 5,000 rpm for $5 \mathrm{~min}$. The cell sediment was washed three times with fresh $\mathrm{M}-\mathrm{H}$ broth medium and then fixed with $2.5 \%$ glutaraldehyde in phosphate buffer ( $\mathrm{pH}$ 7.2) at $4{ }^{\circ} \mathrm{C}$ for $1 \mathrm{~h}$, washed three times with phosphate buffer for $10 \mathrm{~min}$ and fixed again with $1 \%$ osmium tetroxide for $2 \mathrm{~h}$. This was followed by three washings in phosphate buffer for $10 \mathrm{~min}$ and subsequently dehydrated in a series of ethanol concentrations (30, 50, 70, 90 and 95\%), for $15 \mathrm{~min}$ each. The samples were subjected to $100 \%$ ethanol and $\mathrm{CO}_{2}$ to achieve the critical point and then coated with gold ion in a pressure metallic chamber. At the end of the process, the samples were submitted for analysis by scanning electron microscope (SEM) (Quanta 400, FEI, Czech Republic).

\subsection{Release of UV Absorbing-Materials $260 \mathrm{~nm}$ Assay}

The release of UV absorbing-materials was measured using spectrophotometer (NanoDrop 2000, Thermo Scientific, USA) which was modified from previously published protocol [9]. Supernatant from previous process was immediately filtered through a $0.45 \mu \mathrm{m}$ pore-size filter (Millipore, Germany) to remove residual bacteria cells. The absorbance reading at $260 \mathrm{~nm}\left(\mathrm{~A}_{260}\right)$ from clear supernatant was recorded.

\subsection{Cytotoxicity Assay}

Cytotoxicity of bioactive compounds was 
investigated against the mature brine shrimp, Artemia salina, in one day in vivo according to published protocol [10]. The mature brine shrimp(s) were obtained by hatching brine shrimp eggs which were kindly obtained from the faculty of Aquaculture Technology, School of Agricultural Technology, Walailak University, Nakhon Si Thammarat, Thailand, in artificial sea water (3.8\% sodium chloride solution) at $25{ }^{\circ} \mathrm{C}$ for $48 \mathrm{~h}$. Meanwhile, bioactive compounds were dissolved to prepare as a stock solution at a concentration of $10 \mathrm{mg} / \mathrm{mL}$ of dimethyl sulfoxide (DMSO). The stock solution was introduced into the vials by pipetting at different volumes to prepare for the serially different concentrations of 250.00, 125.00, 62.50, 31.25, 15.62 and $7.81 \mu \mathrm{g} / \mathrm{mL}$. The vial that contained the DMSO without bioactive compounds was designed as a control. Both experimental vials and control vial were added the artificial sea water up to $5 \mathrm{~mL}$ and then transferred 10 mature brine shrimp to all experimental vials and control vial. After incubation at $25{ }^{\circ} \mathrm{C}$ for $24 \mathrm{~h}$, the number of mature brine shrimp was counted. The findings were graphically presented by plotting the concentrations of bioactive compounds versus percentages of the mortality rate of the mature brine shrimp from which $\mathrm{LC}_{50}$ (50\% lethal concentration) was determined by extrapolation.

\subsection{Statistical Analysis}

All the above assays were performed in triplicate and repeated independently three times, for consistency of results and statistical purpose. The obtained data were analyzed by using the Statistical Package for the Social Sciences (SPSS) software version 17. One-way ANOVA (Analysis of Variance) was employed and the level of significance was $p<$ 0.05 . Post-hoc turkey analysis was used to investigate the differences between the data.

\section{Results and Discussions}

Rapidly increasing of MRSA infections has become issues of very important public health concern. Although, intravenous vancomycin remains the standard therapy, but concerns about MSSA strains with reduced sensitivity to vancomycin and about the resistant increase to vancomycin of MRSA were remained. Therefore, the screenings of anti-MRSA compounds were continuously conducted for the development of new antibiotic. Fortunately, the bioactive compounds from producing strain of Streptomyces sp. KB1 had anti-MRSA activity when observed by agar well diffusion method. Its activity was remained when treated with hotness at $60{ }^{\circ} \mathrm{C}$ for $30 \mathrm{~min}$.

\subsection{Mechanism of Action of Bioactive Compounds}

MRSA 2468 was cultured in M-H broth medium at $37{ }^{\circ} \mathrm{C}$ until mid-log phase, supplemented with bioactive compounds and continuously cultured at same condition as described in Section 2. Every $8 \mathrm{~h}$,
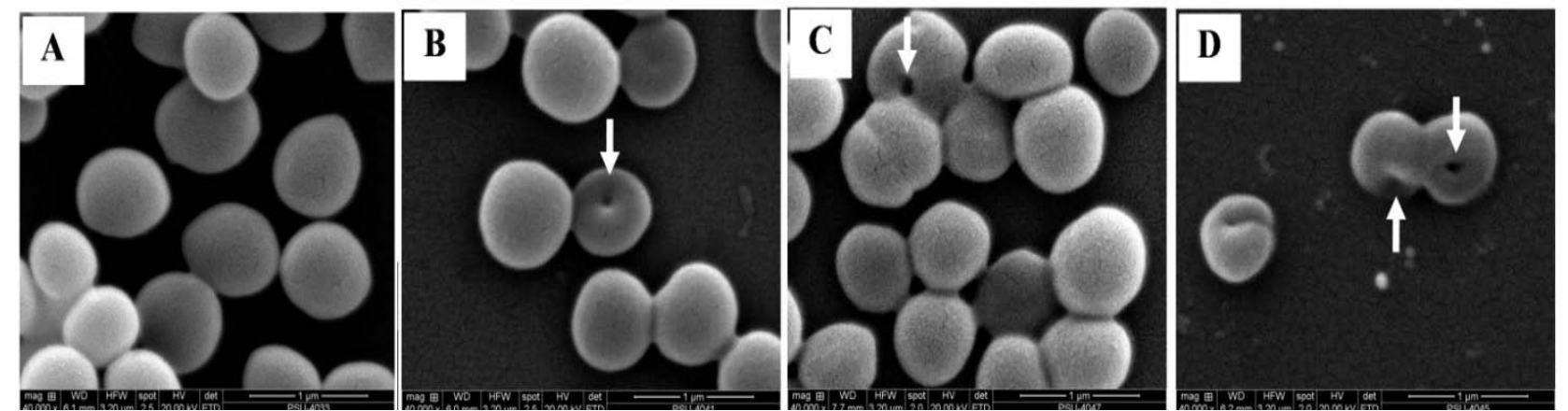

Fig. 1 (A) The SEM photograph of normal cells of MRSA 2468, (B, C and D) cells of MRSA 2468 after exposing with 31.25 $\mu \mathrm{g} / \mathrm{mL}$ of bioactive compounds (MIC-value) for 8, 16 and $24 \mathrm{~h}$ respectively. The arrow head indicated the bursting cell at cell wall of cell treated with bioactive compounds. 
Table 1 Concentration of $260 \mathrm{~nm}$ absorbing-materials in supernatant obtained from cultivating MRSA with or without bioactive compounds at different incubation periods.

\begin{tabular}{llll}
\hline \multirow{2}{*}{ Supernatant from cultivating MRSA } & \multicolumn{3}{c}{ Genetic materials concentration (ng/mL) in supernatant at } \\
\cline { 2 - 4 } & $8 \mathrm{~h}$ & $16 \mathrm{~h}$ & $24 \mathrm{~h}$ \\
\hline Without bioactive compounds & $\mathrm{ND}^{*}$ & $\mathrm{ND}^{*}$ & $\mathrm{ND}^{*}$ \\
With $31.25 \mu \mathrm{g} / \mathrm{mL}$ of bioactive compounds & 1.2 & 17.4 & 36.3 \\
\hline
\end{tabular}

${ }^{*}$ Not determined, it was assigned as blank.

MRSA, methicillin resistant Staphylococcus aureus.

MRSA cells were harvested and observed cell feature by SEM. From the SEM photograph (Fig. 1), the deformation of MRSA cells was observed. The mode of action of bioactive compounds is clear whose major target was cell wall. For the damage confirmation of the cell wall of MRSA, the supernatant was harvested to determine the genetic material in the later time.

\subsection{Concentration of $260 \mathrm{~nm}$ Absorbing-Materials}

The bacterial cell wall is an extremely important component of the cell. The damage of them is usually associated with cell death. Therefore, the most bioactive compounds that have target site at here often show bactericidal or fungicidal activity [11]. It corresponds with our research which was found that bioactive compounds cause the damage of cell wall of MRSA and also showed bactericidal activity. The damage of the cell wall of MRSA was confirmed by genetic materials which were measured from the culture broth of them at $260 \mathrm{~nm}$. The results presented in Table 1, indicated that genetic materials (DNA and RNA) were released. The release of intracellular components is a good indicator for integrating of the bacterial cell wall. If it is damaged, small ions such as potassium and phosphate tend to leach out first, followed by large molecules such as DNA, RNA, and other materials. Since these nucleotides have strong UV absorption at $260 \mathrm{~nm}$, they are defined as " $260 \mathrm{~nm}$ absorbing-materials" [12]. In this study, it is clear that bioactive compounds have the mode of action same as vancomycin. However, it is possible that the mechanism of action of bioactive compounds differs with vancomycin which we will further study in the future.

\subsection{Cytotoxicity against the Mature Brine Shrimp}

The cytotoxicity of bioactive compounds was evaluated by brine shrimp lethality bioassay. The obtained data found that the effect of bioactive compounds on the mortality rate of the mature brine shrimp showed cytotoxic activity dependent concentration. After bioactive compounds concentration versus the percentage of the mortality rate of the mature brine shrimp was plotted as a linear correlation graph and constructed as the linear equation, it could interpret the result of $\mathrm{LC}_{50}$ value as $64.85 \mu \mathrm{g} / \mathrm{mL}$. The obtained result demonstrated that bioactive compounds have moderate cytotoxic activity. When comparing with Minimum Inhibition Concentration (MIC) value, it was found that $\mathrm{LC}_{50}$ value remained significantly higher than the MIC value $(31.25 \mu \mathrm{g} / \mathrm{mL})(p<0.05)$. For determination of the cytotoxic activity of bioactive compounds, several methods are employed. A widely used method in this field is brine shrimp lethality bioassay because it is a rapid method utilizing only $24 \mathrm{~h}$, inexpensive, and needs no special equipment. Moreover, the interest of this method was the use of zoological organism to investigate the cytotoxic activity [13]. In this research, brine shrimp lethality bioassay was used to preliminarily assess the cytotoxicity of bioactive compounds because animal cytotoxicity test shows the variations depending on the chemical nature of the compound and the route of administration to the experimental animal [14]. Therefore, in vivo cytotoxicity study in rat along with the pharmacodynamics and pharmacokinetics will be performed in the next time. 


\section{Conclusion}

Based on the results of this study, it could be concluded that bioactive compounds which were produced by Streptomyces sp. KB1 TISTR 2304 had the potent anti-MRSA activity and the mode of action at the cell wall and also showed the moderate cytotoxic activity. All obtained results implied that bioactive compounds can be used as a model for novel anti-MRSA drug development. Therefore, future works will investigate the mechanism of action, and confirm the in vivo cytotoxicity in rat, along with studying the pharmacodynamics and pharmacokinetics of this compound.

\section{Acknowledgments}

The research was supported by Walailak University, Individual Research Grant (WU-IRG-62-021).

\section{References}

[1] Schito, G. C. 2006. "The Importance of the Development of Antibiotic Resistance in Staphylococcus aureus." Clinical Microbiology and Infection 12: 3-8.

[2] Montazeri, A. E., Khosravi, A. D., Feizabadi, M. M., Goodarzi, H., Khoramrooz, S. S., Mirzaii, M., Kalantar, E., and Sarokhalil, D. D. 2013. "The Prevalence of Methicillin-Resistant S. aureus (MRSA) Isolates with High-Level Mupirocin Resistance from Patients and Personnel in a Burn Center.” Burns 39: 650-54.

[3] Oancea, S., and Stoia, M. 2010. "Antibiotic Resistance of Bacterial Pathogens: The Magnitude of the Problem from Two Perspectives-Romanian and Worldwide.” Romanian Biotechnological Letters 15: 5519-29.

[4] Deurenberg, R. H., Vink, C., Kalenic, S., Friedrich, A. W., Bruggeman, C. A., and Stobberingh, E. E. 2007. "The Molecular Evolution of Methicillin-Resistant Staphylococcus aureus." Clinical Microbiology and Infection 13: 222-35.

[5] Chang, S., Sievert, D. M., Hageman, J. C., Boulton, M. L., Tenover, F. C., Downes, F. P., Shah, S., Rudrik, J. T., Pupp, G. R., Brown, W. J., Cardo, D., and Fridkin, S. K.
2003. "Infection with Vancomycin-Resistant Staphylococcus aureus Containing the vanA Resistance Gene.” Journal of Medicinal Chemistry 348: 1342-7.

[6] Bush, K. 1997. “Antimicrobial Agents.” Current Opinion in Chemical Biology 1: 169-75.

[7] Rahman, M. A., Islam, M. Z., Khondkar, P., and Islam, M. A. 2010. "Characterization and Antimicrobial Activities of a Polypeptide Antibiotic Isolated from a New Strain of Streptomyces parvulus.” Bangladesh Pharmaceutical Journal 13: 14-6.

[8] Kamonwannasit, S., Nantapong, N., Kumkrai, P., Luecha, P., Kupittayanant, S., and Chudapongse, N. 2013. “Antibacterial Activity of Aquilaria crassna Leaf Extract against Staphylococcus epidermidis by Disruption of Cell Wall.” Annals of Clinical Microbiology and Antimicrobials 1: 12-20.

[9] Ahmad, S. J., Lian, H. H., Basri, D. F., and Zin, N. M. 2015. "Mode of Action of Endophytic Streptomyces sp. SUK 25 Extract against MRSA; Microscopic, Biochemical and Time-Kill Analysis.” International Journal of Pharmaceutical Sciences Review and Research 30: 11-7.

[10] Sivasankar, P., Manivasagan, P., Vijayanand, P., Sivakumar, K., Sugesh, S., Poongodi, S., Maharani, V., Vijayalakshmi, S., and Balasubramanian, T. 2013. "Antibacterial and Brine Shrimp Lethality Effect of Marine Actinobacterium Streptomyces sp. CAS72 against Human Pathogenic Bacteria." Asian Pacific Journal of Tropical Disease 3: 286-93.

[11] Ho, P. L., Lo, P. Y., Chow, K. H., Lau, E. H., Lai, E. L., Cheng, V. C., and Kao, R. Y. 2010. "Vancomycin MIC Creep in MRSA Isolates from 1997 to 2008 in a Health Care Region in Hong Kong.” Journal of Infection 60: 140-5.

[12] Allen, N. E., and Nicas, T. I. 2003. "Mechanism of Action of Oritavancin and Related Glycopeptide Antibiotics.” FEMS Microbiology Reviews 26: 511-32.

[13] Sudha Kesavan, S., Vijayalakshmi, S., Nandhini, S. U., Latha, M. B., and Selvam, M. M. 2011. “Application of Brine Shrimp Bioassay for Screening Cytotoxic Actinomycetes.” International Journal of Pharmacy and Pharmaceutical Science Research 1: 104-7.

[14] Roy, R. N., Laskar, S., and Sen, S. K. 2006. "Dibutyl Phthalate, the Bioactive Compound Produced by Streptomyces albidoflavus 321.2.” Microbiological Research 161: 121-6. 